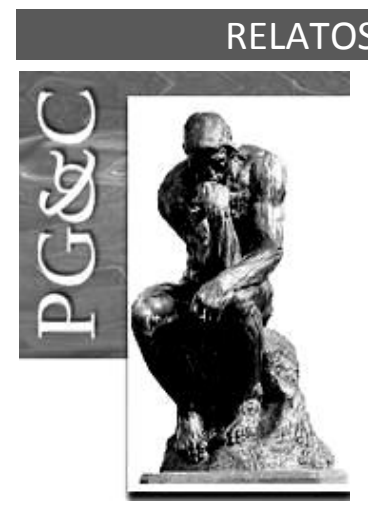

\title{
A INTERCULTURALIDADE E SUA INFLUÊNCIA NA GESTÃO DO CONHECIMENTO: A EXPERIÊNCIA DA VOLVO DO BRASIL
}

\author{
Gislaine Martinelli Baniski \\ Doutora em Administração pela Universidade Positivo, Brasil. Professora \\ da Universidade Estadual de Ponta Grossa, Brasil. \\ E-mal: gislainebaniski@gmail.com \\ Rubens Cieslak \\ Especialista em Recursos Humanos da Volvo do Brasil \\ Email: rubens.cieslak@volvo.com
}

\begin{abstract}
Resumo
Este estudo pretende verificar como a interculturalidade tem influenciado nas práticas de gestão do conhecimento em equipes interculturais. Visando atingir o objetivo proposto, foi realizada uma pesquisa qualitativa, descritiva, que se configurou num estudo de caso. A organização foco do estudo é uma multinacional de origem sueca, fabricante de caminhões médios e pesados, além de equipamentos náuticos e para construção, com subsidiária situada na cidade de Curitiba e com a matriz na cidade de Gottemburgo, na Suécia. Foi realizada a coleta de dados primários por meio de entrevistas presenciais a 27 funcionários, em sua maioria em funções de direção e gerência, e da observação direta na subsidiária no Brasil e na matriz na Suécia. Os resultados demonstraram que as dimensões apresentadas pelos participantes como influentes para os resultados do time são descritas como: a auto-gestão, a autonomia do time, o formato de reconhecimento e feedback, a integração global de dados. O modo como se dava a participação dos membros influenciava a performance da equipe, que era afetada por com aspectos como: Imersão na cultura organizacional, Exposição intercultural, Tempo de casa, Motivação pessoal e de crescimento. Conclui-se que a Gestão Intercultural do Conhecimento envolve a adaptação para promover práticas de conexão do conhecimento, favorecendo resultados organizacionais, em times interculturais.
\end{abstract}

Palavras-chave: interculturalidade, gestão do conhecimento, contexto capacitante.

\section{INTERCULTURALITY AND ITS INFLUENCE IN KNOWLEDGE MANAGEMENT: VOLVO DO BRAZIL'S EXPERIENCE}

\begin{abstract}
This study aims to verify how interculturality has influenced the knowledge management practices in intercultural teams. Aiming to reach the objective, a case study was carried out, using a qualitative, approach. The study was developed in a Swedish multinational manufacturer of medium and heavy trucks, as well as nautical and construction equipment, with a subsidiary located in Curitiba, Brazil and its headquartered in Gottemburg, Sweden. Primary data were collected through face-to-face interviews with 27 employees, mostly in management and management roles, and direct observation in the Brazilian subsidiary and head office in Sweden. The results showed that the dimensions presented by the participants as influential to the results of the team are described as: self-management, team autonomy, recognition and feedback format, global data integration. The participation of the members influenced the performance of the team, which was affected by aspects such as: Immersion in organizational culture, intercultural exhibition, home time, personal motivation and growth. It is concluded that the Intercultural Knowledge Management involves the adaptation to promote practices of connection of knowledge, favoring organizational results, in intercultural teams.
\end{abstract}

Keywords: interculturality. knowledge management. Enabling context.

Perspectivas em Gestão \& Conhecimento, João Pessoa, v. 8, número especial, p. 70-85, out. 2018. DOI: http://dx.doi.org/10.21714/2236-417X2018v8nep70

http://periodicos.ufpb.br/ojs2/index.php/pgc. ISSN: 2236-417X. Publicação sob Licença (cc) EY-Nc-ND 


\section{INTRODUÇÃO}

O ambiente organizacional tem sido influenciado por constantes trocas interculturais, tanto por meio de rotinas como transações com outros países, parcerias, aquisição, processos de expatriação, times multiculturais, como por relações que afetam o próprio indivíduo, como a exposição cultural por meio de viagens, acesso à informação e à tecnologia. Este panorama conduz ao imperativo intercultural (FREITAS, 2008).

A interculturalidade tem sido um fenômeno bastante estudado pois, além da globalização e sua influência econômica, o acesso a informação tornou o mundo mais próximo, culturas diferentes puderam conhecer melhor umas às outras, o que tornou a sociabilidade mais possível, o transporte intercontinental ficou mais acessível e o turismo entre países bastante utilizado (FREITAS, 2008, HAJRO; GIBSON; PUDELKO, 2015); isto gerou aproximação entre diferentes panoramas culturais desencadeando a necessidade de melhor gestão destas trocas e interrelações (RODRIGUES, 2010; HINDS; LIU; LYON, 2011; SCHMIDMEIER, 2016).

A sociedade tem formado arranjos diferenciados se forem consideradas as influências econômicas, tecnológicas e principalmente culturais, assim, delineia-se uma atuação cada vez mais global, por consequência, um mundo corporativo com características mistas e difusas. Autores como Castells (1999) e Bauman (2001) destacam as novas configurações do panorama social atingindo as formatações culturais, em especial no mundo organizacional, como sociedade em rede, conflitos culturais, mobilidade humana e necessidades de adaptação ao novo contexto. Elementos estes que têm desencadeado o interesse em pesquisas e estudos mais aprofundados (HAJRO; GIBSON; PUDELKO, 2015; RODRIGUES, 2010; SCHMIDMEIER, 2016).

Tanto as pessoas como as organizações desenvolvem necessidades que fazem com que a competência em lidar com ambientes multiculturais esteja presente no cotidiano, exigindo dos profissionais talento para a mobilidade, comunicação eficiente e adequação cultural.

A pertinência de se considerar a cultura na compreensão da gestão além-fronteiras se justifica ao se notar que estes elementos estão alterando as práticas sociais, as crenças compartilhadas estão recebendo influência de clientes, fornecedores e parceiros de outros contextos, o que tem afetado a forma como o cotidiano empresarial tem se reorganizado. A presença de times interculturais no cotidiano corporativo ratifica a importância de se compreender como gerenciar estes elementos de modo a adicionar mais valor e eficiência a estas interações, gerando produtividade, criatividade e inovação (BANISKI, 2018).

Neste cenário, times interculturais têm se dedicado à criação conjunta de inovações onde a criação e o compartilhamento do conhecimento tornam-se requisitos necessários. Ocorre, entretanto, que diferenças culturais podem diminuir a eficiência de tais equipes, caracterizando-se em barreiras, como por exemplo, a conversação e argumentação num idioma que não é o nativo, a compreensão de ideias oriundas de percepções culturais distintas, além de uma possível falta de integração e convivência. Outrossim, estas distintas visões podem se traduzir na ampliação da criatividade e de múltiplas adições a um projeto. Assim, a gestão do conhecimento requer atenção mais próxima, para ser entendida em contextos interculturais.

Pensando em compreender de que modo a interculturalidade tem influenciado nas práticas de gestão do conhecimento foi realizado um estudo em uma multinacional sueca com subsidiária no Brasil, verificando-se as dimensões que impactavam a performance e resultados de times interculturais. Para tanto, optou-se por uma pesquisa qualitativa, descritiva abordada na forma de um estudo de caso. Os dados foram coletados por meio de entrevista pessoal, análise documental e observação direta e compunham de modo completo a tese de doutorado

Perspectivas em Gestão \& Conhecimento, João Pessoa, v. 8, número especial, p. 70-85, out. 2018. 
da autora, realizada no ano de 2018. Para este artigo são apresentados apenas aspectos relacionados ao impacto da interculturalidade na gestão do conhecimento.

Inicialmente são resgatados aspectos teóricos relacionados à interculturalidade nas organizações e como esta tem se relacionado com a gestão do conhecimento na literatura. $\mathrm{Na}$ sequência são explanados os procedimentos metodológicos e a análise das dimensões que configuraram como influentes na gestão do conhecimento. Um modelo é apresentado como síntese destas dimensões, seguindo-se para as conclusões e recomendações de estudos futuros.

\section{A INTERCULTURALIDADE NAS ORGANIZAÇÕES}

A interculturalidade abordada na concepção dos gestores de multinacionais ou transnacionais tem um sentido diferente do de multiculturalismo, este com uma conotação mais ligada a movimentos sociais, que visam o reconhecimento das diferenças nas identidades pessoais, diversidade cultural e étnica, não estando relacionados a integração ou objetivando unir a multiculturalidade e sim manter a diferença. Já a interculturalidade julga ser necessária uma comunicação comum visando a convivência e compreensão mútua dos envolvidos (BARBOSA; VELOSO, 2007).

Visando esclarecer a diferença entre multicultural, intercultural e transcultural, a multiculturalidade tem sido adotada para indicar a convivência entre diferentes grupos culturais num mesmo contexto social, já a interculturalidade traz uma concepção de perspectivas distintas entre grupos sociais, trazendo a singularidade de cada grupo, já a transculturalidade pode ser entendida como o produto original da hibridização de elementos culturalmente diferentes. A interculturalidade pressupõe interação, que se produz com e sobre cada um dos parceiros da percepção, ação e relação cooperativa ou conflituosa (FREITAS, 2008; HINDS; LIU; LYON, 2011).

Adotou-se nesta pesquisa a definição de interculturalidade como sendo a interação de diferentes culturas, que é representada pela adoção de uma comunicação comum, trocas mútuas em relações igualitárias, visando a convivência e compreensão entre os envolvidos com finalidade de atingir objetivos comuns (BARBOSA, VELOSO, 2007; DAMÁZIO, 2008; FREITAS, 2008; TAYLOR; OSLAND, 2011).

A cultura corporativa influencia diretamente na forma como uma empresa responde aos estímulos tanto internos como externos. Mesmo pelo julgamento dos elementos expressos como artefatos, rituais, mitos que os grupos apreenderam como forma de lidar com a adaptação interna ou externa (SCHEIN, 2009), a cultura organizacional requer um olhar mais aprofundado dos discursos expressos pelos atores organizacionais.

A interculturalidade não obteve foco de análise desde o começo das pesquisas sobre cultura e nem sempre sua compreensão era desejada. Cuche (1999) afirma que poucos trabalhos estudaram o processo de mudança cultural ligados a contatos culturais, principalmente devido à "superstição do primitivo", um mito de que era importante estudar as culturas primitivas, consideradas puras, por isto a mestiçagem entre as culturas não era vista com bons olhos. Durkheim considerava que dois sistemas sociais e culturais são diferentes e que não podia haver interpenetração entre eles, conforme Cuche (1999).

Após um tempo, a aculturação passou a ser então vista como uma evolução natural da cultura e perdeu um pouco de sua interpretação pejorativa, a interculturalidade foi mais associada a processos de trocais culturais ligadas principalmente à imigração e à miscigenação da sociedade.

A observação da construção de uma cultura deve ser acompanhada pelo fato que o funcionário incorpora ou é incorporado pela atmosfera predominante na empresa sem que possa perceber ou tomar uma posição, por isso, Schein (2009) alerta que é especialmente

Perspectivas em Gestão \& Conhecimento, João Pessoa, v. 8, número especial, p. 70-85, out. 2018. 
perigoso tentar inferir suposições mais profundas apenas de artefatos, porque as interpretações de alguém inevitavelmente trazem projeções de seus sentimentos e reações. 0 tempo de observação e profundidade permitem tornar o entendimento dos artefatos mais claro. O principal argumento deste autor é que, a menos que se chegue ao nível das suposições básicas, não é possível decifrar os artefatos, os valores e as normas de um grupo. Por outro lado, quando se encontram algumas dessas suposições básicas e se examina seu inter-relacionamento, pode-se realmente chegar à essência de uma cultura e explicar muito do que ocorre no seu interior. Às vezes, essa essência pode ser analisada como o paradigma em que algumas organizações funcionam em virtude de um conjunto de suposições coordenadas e interconectadas.

O intercâmbio entre padrões diferentes de pensar e agir pode influenciar os pressupostos organizacionais à medida que trocas fazem parte dos relacionamentos interpessoais. Schein (2009, p. 15) esclarece:

\begin{abstract}
O fenômeno social de interação, normalmente norteado pela convivência do ambiente de trabalho, e que, mesmo que espontânea e não sistemática promove, gradualmente, um comportamento dotado de padrões e normas comuns, aceitas e repassadas às pessoas da empresa. Esta movimentação, espontânea é uma forma de construção da cultura, ou seja, a cultura de um grupo é formada contato a contato, pelo convívio e disseminada quando há um novo componente, bem como alterações destes padrões.
\end{abstract}

Assim sendo, alguns pensadores entendem que as culturas corporativas poderiam ser influenciadas e modeladas. A construção intencional das culturas é defendida por estudiosos, como Collins e Porras (1998), que afirmam que culturas, como as de devoção, são essenciais para a longevidade organizacional. Ao esmiuçar que as culturas de devoção envolvem comportamentos e atitudes de doação e comprometimento e ao suscitarem adoração à ideologia central da empresa, o orgulho da reputação e a homogeneização, dotam o líder como o grande maestro da cultural organizacional. Isto exemplifica a cultura organizacional sendo percebida como gerenciável pelo homem.

Existem pesquisadores que contrapõem este aspecto de se moldar uma cultura organizacional em particular. Alvesson (2013) alerta sobre a visão, por vezes problemática, de considerar a cultura como única, como se refletisse mini sociedades. Ele retrata que a multipluralidade e as subculturas de uma organização precisam ser consideradas para melhor compreensão das organizações. É requerida a reflexão sobre quais são os elementos-chave na produção e reprodução das manifestações culturais, quais os maiores direcionadores de forças por traz das crenças compartilhadas.

Deve-se entender que a cultura de uma organização se desenrola numa maneira processual e desenvolve-se através do tempo pelo qual a organização é fundada. As pessoas que compõem a organização engajam-se numa série de interações no ambiente de trabalho, então criam estruturas internas para responder a estas interações. Como resultado, uma identidade organizacional é criada, o que permite aos membros adotarem valores similares e agirem de formas semelhantes escolhendo padrões reconhecidos pela organização, para lidar com questões internas e externas (FREITAS, 2008; HINDS; LIU; LYON, 2011; SCHEIN, 2009).

Estes pressupostos inconscientes determinam como os membros percebem, pensam e sentem, como e por que eles se comportam de um modo em particular e porque certos valores são tidos como certos. Neste sentido, a cultura ou a gestão intercultural, pode ser considerada como uma forma abstrata de interpretar fenômenos ordinários que ocorrem num ambiente, quer seja formalmente construídos ou não. Também deve ser notada a força das

Perspectivas em Gestão \& Conhecimento, João Pessoa, v. 8, número especial, p. 70-85, out. 2018. 
interações sociais, das quais derivam elementos concretos, atitudinais e dotados de persuasão (SCHEIN, 2009).

Nestas interações, considera-se também a troca entre culturas, que ocorre naturalmente no mundo organizacional. Ao atuar na implementação da estratégia, emergem ações coletivas como coalisões e networks, neste sentido diferentes culturas influenciam como as pessoas, empresas e outros agentes relacionam-se uns com os outros (ALVESSON, 2013).

De acordo com Hofstede, (1997) cultura é a programação coletiva da mente que distingue os membros de um grupo de outros. Considerando-se os entrecruzamentos entre distintas culturas nacionais e seus impactos nas organizações, os estudos de Hofstede (1980) ficaram muito conhecidos e até hoje são base para muitos estudos. Este autor desenvolveu pesquisas envolvendo diversos países que, inicialmente desencadearam-se em quatro dimensões culturais a saber: distância do poder, resistência a incerteza, coletivismo e individualismo e masculinidades e feminilidades, posteriormente foi adicionada a visão de longo e curto prazo (HOFSTEDE, 1980) e após novas inserções, incluíram indulgência e restrição, como medidas de como as pessoas aproveitam a vida (HOFSTEDE; HOFSTEDE; MINKOV, 2010). Outra maneira de ver as dimensões da cultura pode ser feita considerando-se os estudos feitos pelo projeto Liderança Global e Efetividade do Comportamento Organizacional - GLOBE, no qual se propõe nove dimensões a serem aplicadas, algumas baseadas em Hofstede (1980), sendo todas elas: distância do poder; aversão à incerteza; coletivismo institucional; coletivismo em grupo; igualitarismo de gênero; assertividade; orientação para o futuro; para o desempenho e orientação humana (HOUSE et al., 2004).

$\mathrm{Na}$ atuação no contexto global, as diferenças culturais podem exercer influência na assimilação, institucionalização e adaptação de práticas de gestão, podendo afetar a performance organizacional (GERHART; FANG, 2005; FREITAS, 2008; REIS, 2014; SCHNECKENBERG; TRUONG; MAZLOOMI, 2015 VICENTE-OLIVA; MARTÍNEZ-SÁNCHEZ; BERGESMURO, 2015).

Estudos têm comprovado que a cultura nacional provê uma importante explicação para a variância na utilização de diferentes práticas, em diferentes países, por outro lado, requer-se cuidado em presumir que a cultura é fator explicativo para elementos não completamente evidenciados (GERHART, 2008). A cultura organizacional que não retrate isomorfismo com a cultura nacional permite maior agência gerencial, permitindo um sistema de gestão mais adaptável à variação intra-país (GERHART, 2008; GERHART; FANG, 2005).

Para ilustrar esta questão de variação intranacional, o Brasil, por ser o maior país em dimensão territorial da América Latina, apresenta claras distinções em sua cultura de norte a sul. Uma empresa que projete instalar-se no Nordeste do país terá impressões culturais bastante diversas de outra que se instale no Rio Grande do Sul. O aporte das funções organizacionais poderá transcorrer diferentemente nestes dois ambientes, mesmo sendo pertencentes a um único país. Assim, pesquisar os traços culturais nas diferentes regiões denota ser um campo interessante para uma compreensão mais detalhada da influência destas culturas.

Uma multinacional americana, implantando operações no Brasil, pode encontrar aderências diferentes de uma empresa japonesa, por exemplo. Algumas nacionalidades podem apresentar pontos convergentes com traços culturais do país a ser implantado, o que pode facilitar a adaptação. Outras culturas podem apresentar pressupostos básicos não sedimentados na cultura local, tornando bastante difícil e longa a trajetória da adaptação cultural. Vale a pena investigar de modo mais refinado esta afirmação.

A diversidade de ambientes nos quais multinacionais operam podem aumentar o número potencial de fontes de inovação e aprendizado. Interações entre indivíduos que possuem diferentes estruturas de conhecimento aumentam a capacidade da organização para

Perspectivas em Gestão \& Conhecimento, João Pessoa, v. 8, número especial, p. 70-85, out. 2018. 
fazer novas ligações, inovando para além do que um indivíduo pode atingir. (EASTERBY-SMITH e LYLES, 2011; SCHNECKENBERG; TRUONG; MAZLOOMI, 2015).

O que se nota é que a habilidade de saber harmonizar a diversidade cultural pode ser um elemento impulsionador valioso na geração de inovação que possa desencadear diferencial competitivo tão desejado no atual cenário de mudanças de expectativas, tanto de clientes, como de funcionários e da sociedade como um todo.

Ainda deve-se considerar que, quando as organizações se envolvem em um processo de globalização do conhecimento local, devem também aplicar a capacidade de ação social, a competência e o desempenho eficaz da tarefa. Mais especificamente, o conhecimento de uma localidade deve gerar vantagem competitivas para outras unidades locais, como a redução de custos de fabricação, o compartilhamento de dados sobre clientes selecionados, a distribuição de um produto comum ou a adoção de programas de treinamento semelhantes (VON KROGH; ICHIJO; NONAKA, 2001).

Empresas multinacionais costumam utilizar-se de grupos interculturais para atuarem em projetos. Os projetos interculturais geralmente são compostos por funcionários de diferentes países, portanto de culturas distintas, o que configura requisitos de agência gerencial (GERHART, 2008; HAJRO; GIBSON; PUDELKO, 2015). Essas práticas são observadas, em seu conjunto, como Gestão Intercultural do Conhecimento, sendo o termo empregado para caracterizar as ações das organizações orientadas para a alinhar a formação de seus funcionários e adaptar práticas, tendo em vista os propósitos organizacionais (BANISKI, 2018).

A atuação com equipes interculturais globais pode representar mais dificuldades de desempenho se comparadas a equipes locais ou virtuais, mas com proximidade física, o gerenciamento destas equipes é mais complexo e os baixos desempenhos podem estar associados a falta de preparo, treinamento e estrutura. As dificuldades em conduzir a eficiência de projetos com equipes globais estão relacionadas à dispersão física, culturas e línguas diferentes e falta de identidade de time, elemento que é mais facilmente desenvolvido em encontros presenciais. (RODRIGUES, 2010).

Entre as vantagens das equipes interculturais estão a variedade de perspectivas, habilidades e atributos pessoais, permitindo abordagens mais criativas, identificando-se melhor um problema e gerando melhores soluções. (HAJRO; GIBSON; PUDELKO, 2015; RODRIGUES, 2010).

Outro elemento que merece atenção com relação à equipes interculturais relaciona-se ao fato que diferentes identidades de membros de um time podem desencadear sentimentos de negatividade e estereótipos, e ações de integração e envolvimento emocional e de valorização pode ser positivamente ligadas ao compartilhamento do conhecimento em projetos interculturais (HAJRO; GIBSON; PUDELKO, 2015; SACKMANN; FRIESL, 2007).

Além disso, Taylor e Osland (2011) afirmam que os elementos de comunicação que tem exercido influência na relação intercultural são: marginalidade, estereótipos, diferenças de estilo, habilidades linguísticas, cosmopolitanismo, sensibilidade cultural, inteligência cultural e a disposição em aceitar algo como satisfatório.

Para obterem sucesso nas relações entre grupos interculturais se faz necessário o desenvolvimento de competências "bi-culturais" que podem favorecer trocas e relacionamentos, facilitando o compartilhamento de informações, em especial por meio da adaptação comportamental das adequações culturais (HONG, 2010), estes resultados podem favorecer entregas mais inovadoras, em especial se envolvidas em círculos de confiança e solicitude (ALAARJ; ABIDIN-MOHAMED; BUSTAMAM, 2016).

A confiança em equipes virtuais pode ser vista como um elemento multidimensional que está relacionado à honestidade, à benevolência e à competência em uma comunidade virtual (CASALÓ; FLAVIÁN; GUINALIU, 2009).

Perspectivas em Gestão \& Conhecimento, João Pessoa, v. 8, número especial, p. 70-85, out. 2018. 
Os estudos supracitados, então, têm confirmado que a habilidade em saber harmonizar a diversidade cultural pode ser um elemento impulsionador valioso na geração de inovação, elemento de grande interesse na gestão das empresas no atual cenário competitivo e dinâmico.

\subsection{Gestão do Conhecimento e interculturalidade}

A capacidade das organizações de explorarem o ambiente de maneira flexível e adaptativa e assim promoverem sua perpetuidade tem sido ligada à condição de criarem e mobilizarem conhecimento transformando-o em inovação e competitividade. Esta mobilização para o uso da informação já era afirmada por Drucker (1993) que citou que a sociedade do conhecimento irá obter da informação e do aprendizado a fonte de diferencial competitivo duradouro.

Esta sociedade deverá fazer uso das informações de modo a transformá-las em conhecimento, sendo assim entendido: Conhecimento é uma mistura fluida de experiência condensada, valores, informação contextual e insight experimentado, a qual proporciona uma estrutura para a avaliação e incorporação de novas experiências e informações (DAVENPORT; PRUSAK, 2003).

Segundo Easterby-Smith; Lyles (2011), o conhecimento é invisível e muitas vezes vem à mente apenas quando precisamos dele para responder a uma pergunta ou resolver um problema; envolve sempre uma pessoa que conhece, baseada numa experiência, uma reflexão que fez sentido. Para estes autores, o conhecimento flui através das comunidades, de uma geração para outra; circula por meio de histórias, encontros casuais de corredores e outras práticas e artefatos informais e indocumentados; permitindo que novos conhecimentos sejam criados nos limites do conhecimento antigo.

O conhecimento tem sido abordado na literatura de formas diversas, desde a que percebe o conhecimento organizacional como "objeto" de tal forma que possa ser claramente entendido, codificado e replicado, inclusive de uma organização para outra. Há também a abordagem do conhecimento como um "processo", afeto pelo grupo social que o constrói e pelo ambiente que pode se facilitador da geração desse conhecimento (NONAKA e TAKEUCHI, 1997; CHOO; ALVARENGA NETO, 2010; ALVARENGA NETO; CHOO, 2010; NONAKA; VON KROGH; VOELPEL, 2006).

Estudos como os de Nonaka e Takeuchi (1997) diferenciaram o conhecimento tácito do explícito, formando um continuum por meio da Conversão do Conhecimento (NONAKA; VON KROGH, 2009) e alavancando debates sobre o uso mais estratégico do conhecimento e da importância do compartilhamento por toda organização.

O conhecimento tácito é altamente pessoal, dificilmente visível e exprimível, não sendo fácil de formalizar como: conclusões, insights, modelos mentais e palpites subjetivos, está enraizado nas ações, experiências, emoções, valores e ideais de um indivíduo. Já o conhecimento explícito é facilmente processado por um computador, transmitido ou armazenado num banco de dados e seu compartilhamento facilitado. Nesse sentido, "a criação de um novo conhecimento envolve tanto ideais quanto ideias" (NONAKA; TAKEUCHI, 1997 p.10).

Para que o conhecimento seja fonte de vantagem competitiva sustentável, os concorrentes devem ter dificuldade para atingir o mesmo nível de custo ou a mesma diferenciação produto/serviço, mediante substituição do conhecimento original por algum conhecimento alternativo (VON KROGH; ICHIJO; NONAKA, 2001). Percebe-se, desta forma que, o conhecimento tácito, social ou individual, é mais difícil de imitar do que o conhecimento explícito, constante em documentos e manuais.

Perspectivas em Gestão \& Conhecimento, João Pessoa, v. 8, número especial, p. 70-85, out. 2018. 
O conhecimento pode ser considerado como o processo humano dinâmico de justificar a crença pessoal com relação a verdade e, tanto informação quanto conhecimento, são específicos ao contexto e relacionais na medida em que dependem da situação e são criados na interação social entre as pessoas (NONAKA; TAKEUCHI, 1997). A interação humana compartilhada num contexto social e histórico conduz à construção de uma realidade que irá afetar suas atitudes, comportamento e decisões (BERGER; LUCKMANN, 1966).

Conforme Choo (1996; 2001), o conhecimento organizacional surge quando os modos de uso da informação estão conectados entre si, para constituir uma rede maior de processos através dos quais a organização constrói significados compartilhados sobre suas ações e identidade; descobre, compartilha e aplica novos conhecimentos; e inicia padrões de ação através da busca, avaliação e seleção de alternativas. As pessoas nas organizações criam sua própria realidade subjetiva, num fluxo de fazer sentido do ambiente, definir, selecionar e reter o conhecimento por meio da interpretação que fazem desta realidade (CHOO, 2001; ALVARENGA NETO, 2005).

Processos de gestão do conhecimento são muitos importantes, pois ajudam as organizações a definir, a selecionar, a organizar, a distribuir e a partilhar a informação retida na memória da organização, gerando sistematicamente o processo de criação do conhecimento fundamental para a inovação (NONAKA; TAKEUCHI, 1997; CHOO; ALVARENGA NETO, 2010;). A mobilização sistemática do conhecimento requer, no mínimo, cinco atividades principais: identificar, criar, armazenar, compartilhar e aplicar o conhecimento (BATISTA, 2012).

O modelo mais conhecido de Gestão do Conhecimento é atribuído a Nonaka e Takeuchi (1997) e relaciona o compartilhamento do conhecimento tácito e explícito no ambiente organizacional. A criação do conhecimento ocorre pelo processo de conversão do conhecimento tácito em explícito por meio de um processo social de validação da verdade, havendo a Socialização, Externalização, Combinação e Internalização (SECI) do conhecimento (NONAKA; TOYAMA, 2005; NONAKA; VON KROGH; VOELPEL, 2006).

Um conceito fortemente ligado à cultura japonesa e introduzido na Gestão do Conhecimento por Nonaka e Konno (1998) é o "Ba", definido pelos autores como o "espaço compartilhado" onde sedimenta-se a base para criação do conhecimento. O termo "Ba" passou por tentativas de adaptação para terminologias como como "comunidades estratégicas do conhecimento" (FAYARD, 2003), ou "contexto capacitante" (ALVARENGA NETO; CHOO, 2011).

O contexto capacitante influencia o fluxo de criação do conhecimento e ocorre em níveis desde o individual até o nível interorganizacional. Os processos envolvidos estão ligados à criação, compartilhamento e uso do conhecimento. Como produto deste interrelacionamento as condições capacitantes são relativas a condições sociais e comportamentais; cognitivas e epistemológicas; sistêmicas e informacionais; e estratégicas e estruturais. Para Nonaka et al (2000) o "Ba" será afetado por interações individuais e coletivas, por meios virtuais ou presenciais, no processo de criação do conhecimento.

Desta forma, estudiosos têm afirmado que não se gerencia o conhecimento, mas sim, gerencia-se o ambiente capacitante gerador de conhecimento (ALVARENGA NETO; CHOO, 2010). Conforme Nonaka (2007) a Gestão do Conhecimento fica melhor expressa se adotado o conceito de "Gestão Baseada em Conhecimento - Knowledge Based Management".

Fayard, (2003) defende que Comunidades Estratégicas do Conhecimento, sua proposta como versão ocidental do Ba, promovem projetos colaborativos, inclusive envolvendo outros países. Os parceiros, e mesmo os concorrentes, os clientes e os usuários, fontes complementares de conhecimento e de competências, são engajados sinergicamente em direção de uma frente de criação de conhecimento operacional. Essa porosidade dinâmica da empresa aparece como uma condição para a agilidade estratégica.

Perspectivas em Gestão \& Conhecimento, João Pessoa, v. 8, número especial, p. 70-85, out. 2018. 
O contexto capacitante se beneficia de cinco Condições Capacitadoras: que seriam Intenção, Autonomia, Flutuação e Caos Criativo, Redundância e Variedade de Requisitos, segundo Nonaka e Takeuchi (1997), e/ou de cinco Capacitadores: Instilar a Visão de Conhecimento, Gerenciar Conversas, Mobilizar os Ativistas do Conhecimento, Criar o Contexto Adequado e Globalizar o Conhecimento Local, segundo Von Krogh, Nonaka e Ichijo (2001). Desta forma, organizações que pretendam gerenciar o contexto facilitador para o conhecimento encontram nestes elementos orientação para ações e estratégias.

Alvarenga Neto, Barbosa e Cendón (2006) propuseram um modelo para contexto capacitante, onde observa-se que os processos relativos ao conhecimento (criação, compartilhamento e uso) nos níveis individuais, grupais, organizacionais e interorganizacionais são impactados pelas condições capacitadoras de: estrutura, sistemas de gestão, condições cognitivas e sociais-comportamentais. Para os autores do framework, as organizações podem gerenciar os elementos componentes da estrutura organizacional, dispor de sistemas de informação, proporcionar um ambiente e capacitação que fomente comportamentos e trocas sociais facilitadoras do compartilhamento e uso do conhecimento; desta forma, as inovações e resultantes da gestão do conhecimento encontram terreno fértil num contexto que as permita frutificar.

A questão da interculturalidade na gestão do conhecimento foi discutida por Nonaka e Takeuchi (1997) ao indagarem se o processo de criação do conhecimento usado por empresas japonesas poderia funcionar fora do Japão e quais ajustes nestas práticas seriam necessários. A distância cultural que empresas japonesas encontram frente às abordagens ocidentais precisam ser consideradas: no ocidente a interação entre o conhecimento tácito e o explícito ocorrem principalmente no nível individual, os esforços vem mais da externalização do líder (como o exemplo de Jack Welch da GE), na cultura japonesa, a criação ocorre mais no nível de grupo. Ainda as práticas ocidentais podem sofrer da "paralisia de análise", valendo-se de muitos dados, documentos e gráficos, ao ponto que a filosofia japonesa confia muito no conhecimento tácito e na intuição e frequente interação. Segundo estes autores, a diversidade fornece uma fonte natural de variedade necessária para o aproveitamento de uma condição capacitadora fora da base da empresa. Os exemplos aplicados por empresas japonesas interagindo em outras culturas mostrou que a abordagem pode ser aplicada em culturas diferentes, requerendo mais esforço nas fases de socialização e externalização, pois as pessoas levam mais tempo para compartilhar o conhecimento tácito, bem como estabelecerem laços de confiança.

O fluxo do conhecimento é destacado também por Zollo e Winter (2002), que defendem uma perspectiva de criação de vantagens organizacionais por meio de práticas, rotinas e processos homogêneos e replicáveis, encontrando na Gestão do Conhecimento o alicerce para a seleção de variáveis, replicação, retenção, estímulo externo e recombinação. Esta estrutura garantiria impacto estratégico, uma vez que os mecanismos de conhecimento tácito são mais difíceis de serem copiados.

Além do ambiente facilitador da criação do conhecimento, outros agentes podem interferir na qualidade das trocas interculturais, como a língua nacional utilizada nas comunicações.

Enfim, a criação do conhecimento por grupos com diferentes identidades culturais se faz de encontros e diálogos, o compartilhamento do conhecimento tácito é favorecido por laços de proximidade e confiança, estimulados por trabalhos em grupo e norteados por insights individuais, sempre alinhados às premissas e diretrizes corporativas.

Perspectivas em Gestão \& Conhecimento, João Pessoa, v. 8, número especial, p. 70-85, out. 2018. 


\section{METODOLOGIA}

Para explorar e conhecer a temática pretendida foi realizado um estudo de caso, por meio de uma abordagem qualitativa, descritiva. A abordagem qualitativa se justifica na intenção de entender como o outro percebe os mesmos aspectos, ou outros olhares que interpretam o mesmo elemento. "Os pesquisadores estudam os fenômenos em seus cenários naturais, tentando entender ou interpretar os significados que as pessoas a eles conferem" (DENZIN; LINCOLN, 2006, p17).

A competência da pesquisa qualitativa é, portanto, o mundo da experiência vivida, pois é nele que a crença individual, a ação e a cultura entrecruzam-se, ressaltando a natureza socialmente construída do que é estudado (DENZIN; LINCOLN, 2006).

Por delimitar claramente o objeto de estudo, permitindo uma ampla variedade de evidências foi adotado o Estudo de Caso (STAKE, 2011). O uso de caso único se caracteriza quando o foco de interesse está no individual, no específico, em suas idiossincrasias e complexidades, havendo uma descrição holística e intensiva de um fenômeno bem delimitado, visando a compreensão dos processos sociais, mais que a relação de variáveis (STAKE, 2011), no qual se dá o destaque o caráter particularista e descritivo.

A empresa foco do estudo de caso foi escolhida atendendo aos seguintes critérios: possuía um sistema de gestão do conhecimento institucionalizado; atuava com grupos interculturais em suas rotinas; apresentava inovações desenvolvidas como resultados de grupos interculturais e permitia acesso ao campo para coleta de dados. Desta forma, o estudo de caso foi realizado na Volvo do Brasil, empresa multinacional de origem sueca, com mais de 90 anos de existência, com uma subsidiária com mais de 40 anos situada na cidade de Curitiba, no Brasil. O grupo Volvo tem seu portfólio de marcas voltado para caminhões médios e pesados, equipamentos náuticos e para construção.

Ainda, a Volvo do Brasil é destacada por adotar práticas de gestão inovadoras e com resultados superiores, constando da lista das "melhores empresas para se trabalhar no Brasil", em pesquisa publicada pela editora Abril (revista Exame).

Por ser uma empresa sediada em um país escandinavo, com cultura nórdica, demonstra representatividade de dimensões culturais aderidas tanto à cultura nacional sueca, quanto à identidade organizacional defendida pelo Grupo Volvo, conhecido por sua cultura forte, cuja cultura é alinhada em toda corporação por meio do "The Volvo Way", um manual que concentra e alinha a visão, valores e padrões defendidos pela empresa, que foi atualizado para "Our Way Forward". Além disso, suas práticas de gestão são mundialmente estabelecidas, o modelo de gestão do conhecimento é institucionalizado e as rotinas de desenvolvimento tecnológico são assimiladas por toda a organização.

A coleta de dados primários foi realizada por meio de entrevistas presencias semiestruturadas com 27 funcionários das unidades do Brasil e da matriz na Suécia. Os respondentes foram selecionados de acordo com sua experiência em projetos interculturais ou expatriação, tempo de empresa e atuação em função de gerência ou direção.

Para condução das entrevistas foi adotado um roteiro de entrevista que guiou os pressupostos mínimos de investigação e a coleta de dados foi realizada entre junho e agosto de 2017 para as entrevistas da Suécia e agosto de 2017 a março de 2018 para entrevistas na Volvo do Brasil.

Ainda foi realizado o procedimento de observação direta não-participante (YIN, 1993) em visita técnica presencial nas unidades de Curitiba e na matriz em Gottemburgo, na Suécia, na participação a workshops, eventos, memorial, museus, reuniões interculturais e, em especial, para verificação das características do contexto capacitante da Volvo do Brasil

Os dados secundários caracterizaram-se por manuais, whitebooks, registros internos, relatórios e reportagens públicas. Procedeu-se a coleta até que se caracterizou a saturação dos dados, procedendo-se para a análise de conteúdo, conforme Bardin (2010), sendo adotadas as

Perspectivas em Gestão \& Conhecimento, João Pessoa, v. 8, número especial, p. 70-85, out. 2018. 
etapas de pré-análise, exploração do material, tratamento dos resultados, inferência e interpretação.

\section{RESULTADOS}

Visando apreender os aspectos relativos às práticas de gestão do conhecimento e como estas sofriam influência da interculturalidade nos resultados de equipes de projetos, foram acompanhadas as atividades de grupos interculturais, dentre os quais, mereceu destaque o time que culminou com um produto inovador denominado "VM Autônomo".

Um time intercultural agrega relações em diversas unidades organizacionais e diferentes unidades de negócio (caminhões, motores náuticos) além de parceiros de conhecimento externos à empresa. O compartilhamento do conhecimento entre diferentes indivíduos foi transcorrendo, segundo relatos de entrevistados e análise de evidências, sendo modelado pela cultura natural da empresa, o jeito Volvo (the Volvo Way).

Além das trocas entre os indivíduos, os sistemas de informação da companhia davam um robusto suporte à composição e tomada de decisão nos projetos. No projeto VM Autônomo houve também a participação de um cliente do Brasil.

$\mathrm{Na}$ Volvo, há um repositório que mantém informações das rotinas da empresa e as garantias dos processos que funciona de modo integrado, como descrito pelo responsável pelo sistema: "O SGV é o nosso repositório de documentos Volvo, nasceu para atender a ISO e hoje é uma ferramenta de suporte à gestão e está evoluindo para uma plataforma que concentra e conecta outros portais, tudo está integrado". (E18).

Neste sentido, a codificação do conhecimento (ZOLLO e WINTER, 2002) também permite a rastreabilidade e localização facilitada de informações armazenadas nas diversas unidades da empresa, como citado por um dos entrevistados ligado à área de Engenharia: "[...]temos o Eureka, que é uma espécie de Google Search, que busca conhecimento sobre 'juntas estruturais de chassi' por exemplo, vai aparecer a pessoa na empresa que entende disso, que coloca sua expertise na comunidade de prática [...]". (E16).

Ao articularem o conhecimento interno para responder de modo rápido ao requisitado pelo escopo do projeto manifestaram o domínio das rotinas de modo estável (ZOLLO; WINTER, 2002). Assim, infere-se que a diversidade presente nos grupos interculturais foi imersa no modelo maior em que está envolta a cultura organizacional, permitindo ser afirmado que o contexto capacitante agiu como viabilizador de maior homogeneidade nas relações e nas práticas.

No sentido de levar a organização à maior agilidade, e obter acesso e compartilhamento do conhecimento existente, corrobora-se o uso do termo Gestão Intercultural do Conhecimento, no formato defendido por Baniski (2018), promovendo-se a adaptação cultural e o uso de práticas de conexão que, aliadas ao contexto capacitante, culminaram com resultados inovadores.

As práticas de gestão intercultural do conhecimento estão relacionadas com a competência de resposta do time intercultural por meio de relações afetadas: (a) pelo elevado grau de autonomia atribuída aos membros do projeto, (b) pela integração global (sistema de comunicação integrado) que permite o acesso a informações e conhecimentos de diversas unidades de empresa e (c) pelo formato de reconhecimento coletivo que favorece relações cooperativas entre as unidades e entre os indivíduos.

Seguindo a percepção possibilitada pela análise dos dados, os elementos componentes do contexto capacitante caracterizavam contribuições que ligavam suas relações, de acordo com os entrevistados, conduzindo à conclusão de que os elementos de autonomia, integração dos sistemas e formato de reconhecimento presentes nos grupos interculturais eram impulsionados pelos elementos do contexto. Isto significa que características da cultura

Perspectivas em Gestão \& Conhecimento, João Pessoa, v. 8, número especial, p. 70-85, out. 2018. 
corporativa Volvo, expressas e declaradas em diversos momentos na empresa, são manifestadas nas rotinas de times compondo o contexto capacitante da empresa, características estas como: respeito, solicitude e confiança mereceram destaque, de acordo com dados da pesquisa.

Entretanto, os vínculos de respeito e confiança são mencionados pelos entrevistados como relacionados às entregas e aos combinados realizados no time, como cita um dos entrevistados "confiança na Volvo se faz diariamente, quando se cumpre a palavra, quando se espera algo do outro e se obtém retorno, quando há retribuição e profissionalismo, isso alimenta a confiança e nosso jeito de ser, pois aqui acontece de modo muito espontâneo" (E8). Além disso, os dados das entrevistas permitiram concluir que o perfil do participante dos times interculturais influenciava nos processos, tendo seus resultados afetados pela imersão na cultura organizacional e o tempo de casa, ou seja, indivíduos que vivenciavam o contexto capacitante por mais tempo, adotavam as características atitudinais de respeito e solicitude espontaneamente. A pré-disposição pessoal à experiência intercultural também era associada como um facilitador do trabalho nas equipes à medida que a aceitação e imersão na diversidade agiam como diminuidores de possíveis pontos de conflito.

Com relação às práticas de gestão do conhecimento, evidenciou-se que as práticas que eram associadas à sedimentação das estruturas de confiança e vínculos atinentes ao contexto capacitante eram ações ligadas à memória organizacional, ao uso de comunidades de prática e à adoção de storytelling de modo a sedimentar a cultura cooperativa da empresa. Estas práticas de gestão facilitadoras do compartilhamento do conhecimento foram denominadas de Práticas de Conexão.

Figura 1 - Esquema da Gestão Intercultural do Conhecimento

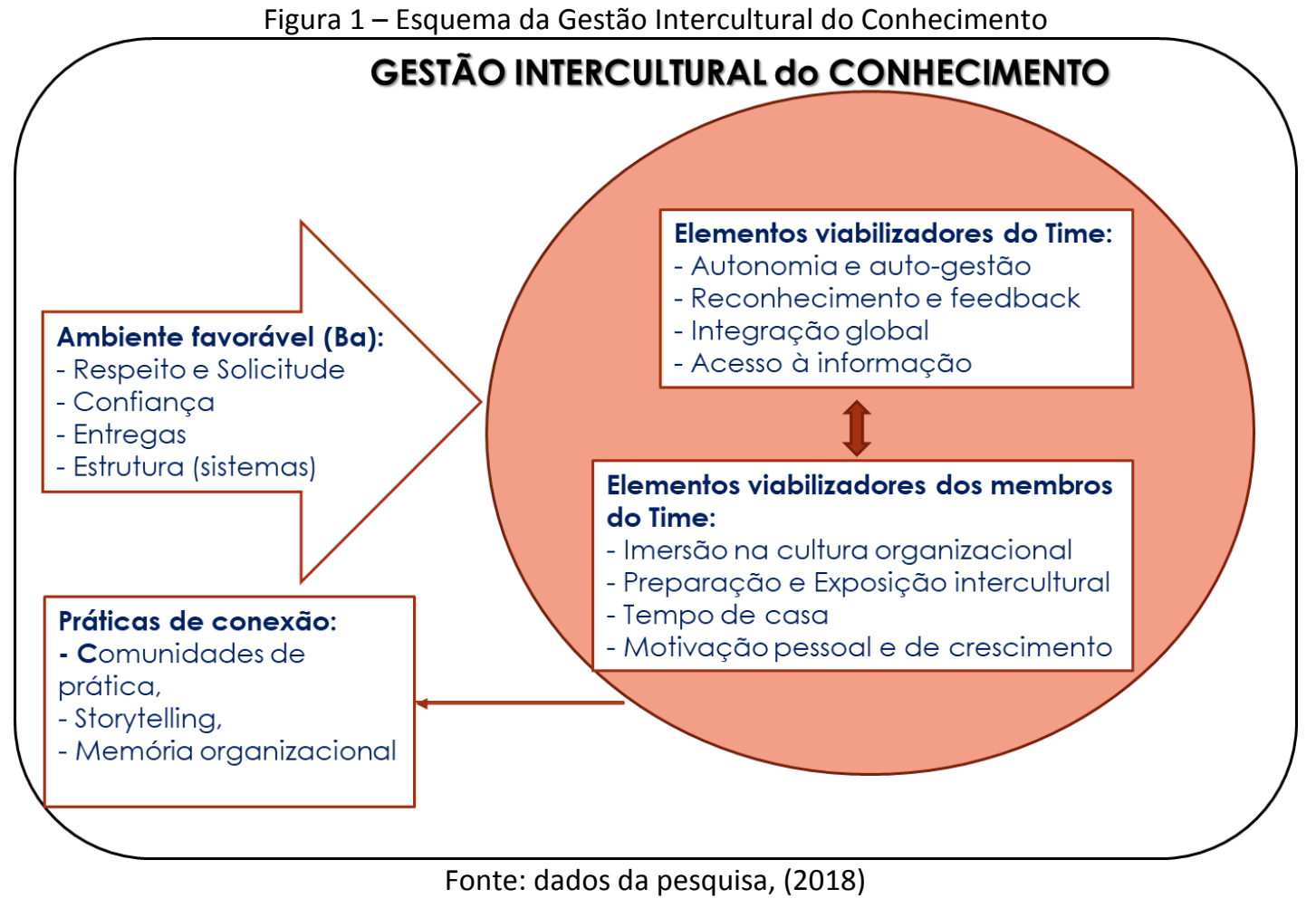

A Figura 1 demonstra de forma esquemática os resultados da pesquisa no sentido de colocar a Gestão Intercultural do Conhecimento como influenciada pelos elementos de gestão do time intercultural (níveis de autonomia, formas de reconhecimento que diminuam a competição e o acesso à informação), alicerçadas por membros de times de projetos

Perspectivas em Gestão \& Conhecimento, João Pessoa, v. 8, número especial, p. 70-85, out. 2018. 
motivados e pré-dispostos para a atuação intercultural. Além disso, a figura 01 destaca o papel definitivo do contexto capacitante em fornecer ambiente favorável à criação e ao compartilhamento do conhecimento.

O contexto capacitante $(\mathrm{Ba})$ Volvo foi associado pelos entrevistados como propício ao respeito e solicitude, que culminam em confiança e atua como favorecedor de entregas e resultados desejados.

As práticas de gestão do conhecimento que mais demonstraram contribuição para com tais resultados, foram nesta pesquisa denominadas de Práticas de Conexão, pois visavam ligar as pessoas a profissionais com domínio em determinado tema, sendo elas: comunidades de prática, memoria organizacional e o uso de storytelling.

\section{CONCLUSÕES}

Com a conclusão deste estudo, foi possível destacar a importância do contexto capacitante como influenciador da atuação da gestão do conhecimento em ambientes interculturais.

$O$ primeiro elemento que pode ser descrito como o fio condutor dos processos analisados na Volvo é o RESPEITO e a CONFIANÇA, presente em uma cultura corporativa que facilita e estimula a criação, compartilhamento e uso do conhecimento. Desta forma, o contexto capacitante Volvo (Ba) cria condições favoráveis para a disseminação de uma cultura para o conhecimento.

Este ambiente de integridade, expresso no manual corporativo "The Volvo Way", enaltece o respeito mútuo que liga as relações de confiança entre as pessoas. Uma vez estabelecidos estes laços de confiança, o compartilhamento e uso do conhecimento alcança uma velocidade maior permitindo resultados e melhorias pretendidos pelas estratégias corporativas.

Quando em interação com culturas diferentes, quer seja clientes ou pessoal interno, a pressuposição básica do "respeito" fica evidente nas atitudes e comportamentos, ditando uma troca relacional balizada pelas concordâncias e não pelas diferenças. Uma vez que a linha de raciocínio esteja voltada para os objetivos comuns que cercam os diversos indivíduos, suas diferenças não são enfocadas, aliás, acabam se tornando irrelevantes, as concordâncias alinham o que as diferenças poderiam afastar.

A estrutura de suporte para que as interações, socialização e compartilhamento ocorram é robusta. Sistemas de informação, repositórios e ferramentas de comunicação imersas nas rotinas e práticas reforçam uma cultura de compartilhamento e dão acesso às informações, facilitando a tomada de decisão. Desde jornais de comunicação interna, newsletters, e-mails que envolvem funcionários, familiares e diversos stakeholders, a comunicação que viabiliza os processos de criação permite trocas com qualquer unidade do globo. Além dos mecanismos virtuais, encontros presenciais são promovidos e estimulados, mas sempre direcionados para o gerenciamento eficiente do tempo e dos resultados.

Equipes internas de projetos interculturais são envoltas na cultura corporativa da confiança e solicitude, o que acaba sendo transferido para o compartilhamento do conhecimento, evitando produção paralela de conhecimento e permitindo que esteja disponível para acesso de toda a organização no globo, o conhecimento de todas as unidades.

Destarte, a denominação de Gestão Intercultural do Conhecimento pretende alinhar a compreensão da importância dos elementos favorecedores de conexão, de modo a criar o ambiente adaptado para a consequente promoção de inovações e resultados organizacionais. O esquema da Gestão Intercultural do Conhecimento (figura 1) reúne as principais dimensões envolvidas na interculturalidade afetando a gestão do conhecimento.

Perspectivas em Gestão \& Conhecimento, João Pessoa, v. 8, número especial, p. 70-85, out. 2018. 
Pesquisas futuras podem averiguar estes elementos em outras empresas, multinacionais de origem brasileira ou estudos multi-caso são fortemente recomendados.

\section{REFERÊNCIAS}

ALAARJ, S.; ABIDIN-MOHAMED, Z.; BUSTAMAM, U. S. B. A. Mediating Role of Trust on the Effects of Knowledge Management Capabilities on Organizational Performance. Procedia Social and Behavioral Sciences, v. 235, p. 729-738, 2016.

ALVARENGA NETO, R. C. D.; CHOO, C.W. 2010. Beyond the Ba: Managing Enabling Contexts in Knowledge Organizations. Journal of Knowledge Management 14(4): 592-610.

ALVARENGA NETO, R. C. D.; BARBOSA, R. R.; CENDÓN, B. V. A construção de metodologia de pesquisa qualitativa com vistas à apreensão da realidade organizacional brasileira. Informação \&Sociedade, v. 16, n. 2, p. 63-78, 2006.

ALVESSON, M. Understanding Organizational Culture. 2nd. ed. Los Angeles: SAGE, 2013.

BANISKI, G. Gestão Intercultural do Conhecimento e Capacidades Dinâmicas: uma proposta conceitual para competência de resposta. 2018. $210 \mathrm{f}$. Tese (Programa de Mestrado e Doutorado da Universidade Positivo) - Universidade Positivo, Curitiba-PR, 2018.

BARBOSA, L.; VELOSO, L. Diferença e Mediação nas Empresas Transnacionais. Civitas - Revista de Ciências Sociais, v. 7, p. 59-85, 2007.

BARDIN, L. Análise de Conteúdo. 4. ed. Lisboa: Edições 70, 2010.

BATISTA, F. F. Modelo de Gestão do Conhecimento para a Administração Pública Brasileira: Como implementar a Gestão do Conhecimento para produzir resultados em benefício do cidadão. Brasilia: IPEA, 2012

BAUMAN, Z. Modernidade líquida. Rio de Janeiro: Jorge Zahar Ed., 2001.

BERGER, P.L.; LUCKMANN, T. A Construção Social da Realidade. 13. ed. Petrópolis: Vozes, 1996.

CASALÓ, L. V; FLAVIÁN, C.; GUINALIU, M. The Role of Trust, Satisfaction, and Communication in the Development of Participation in Virtual Communities. In: AL, C. C. ET (Ed.). Connectivity and Knowledge Management in Virtual Organisations. NY: IGI Global, 2009. p. 188-202.

CASTELLS, M A Era da Informação: economia, sociedade e cultura. A Sociedade em Rede. São Paulo: Paz e Terra, v.1. 1999.

$\mathrm{CHOO}, \mathrm{C}$. W. The knowing organization: How organizations use information to construct meaning, create knowledge, and make decisions. International Journal of Information Management, Vol. 16, No. 5, v. 16 n.5, n. August, p. 329 a 340, 1996.

COLLINS, J. C.; PORRAS, J. Feitas para Durar. Ged. Rio Janeiro: Editora Rocco, 1998.

CUCHE, D. A noção de cultura nas ciências sociais. 2nd. ed. Bauru: EDUSC, 2012.

DAMÁZIO, E. DA S. P. Multiculturalismo versus Interculturalismo: por uma proposta intercultural do Direito. Desenvolvimento em questão, n. 12, p. 63-86, 2008.

DAVENPORT, T. H.; PRUSAK, L. Conhecimento Empresarial: Como as Organizações gerenciam o seu capital. 10. ed. Rio de Janeiro: Elsevier, 2003.

Perspectivas em Gestão \& Conhecimento, João Pessoa, v. 8, número especial, p. 70-85, out. 2018. 
DENZIN, N. K.; LINCOLN, Y. S. O planejamento da pesquisa qualitativa: teorias e abordagens. 2 ed. Porto Alegre: Bookman e Artmed, 2006.

DRUCKER, P. Sociedade Pós-Capitalista. São Paulo: Pioneira, 1993.

EASTERBY-Smith, M. P. V.; LYLES, M. (Eds.) Handbook of organizational learning and knowledge management. (2nd ed. ed.) Chichester: Wiley, 2011.

FAYARD, P. Comunidades Estratégicas de Conhecimento: uma proposta ocidental para o conceito japonês de Ba. 2003

FLEURY, A; FLEURY, M. T. L. Estratégias Empresariais e Formação de Competências: quebracabeça Caleidoscópico da Ind. Bras.3ed. São Paulo: Atlas, 2004.

FREITAS, M. E. DE. O Imperativo Intercultural na Vida e na Gestão Contemporânea. Organizações e Sociedade, v. 15, n. 45, p. 79-89, 2008.

GERHART, B. Cross Cultural Management Research: Assumptions, Evidence and Suggested Directions. International Journal of Cross Cultural Management, v. 8, n. 3, p. 259-274, 2008.

GERHART, B.; FANG, M. National culture and human resource management: Assumptions and evidence. The International Journal of Human Resource, v. 16, n. 6, p. 971-987, 2005.

HAJRO, A.; GIBSON, C. B.; PUDELKO, M. Knowledge exchange processes in multicultural teams: Linking organizational diversity climates to teams' effectiveness. Academy of Management Journal, v. 60, n. 1, p. Advance online publication, 2015.

HINDS, P.; LIU, L.; LYON, J. Putting the global in global work: An intercultural lens on the practice of cross-national collaboration. Academy of Management Annals, v. 5, n. 1, p. 135188,2011

HOFSTEDE, G. Culture's consequences: International differences in work-related values. Beverly Hills, CA: SAGE, 1980.

HOFSTEDE, G. Cultures and Organizations: Software of the Mind. London: McGraw-Hill., 1997.

HOFSTEDE, G.; HOFSTEDE, G. J.; MINKOV, M. Cultures and Organizations: Software of the Mind. 3rd ed. New York: McGraw-Hill., 2010.

HONG, H. J. Bicultural competence and its impact on team effectiveness. International Journal of Cross Cultural Management, v. 10, n. 1, p. 93-120, 2010.

HOUSE, R. et al. Culture, leadership, and organizations: the GLOBE study of 62 societies. Thousand Oaks: Sage, 2004.

NONAKA, I.; TOYAMA, R \& KONNO, N. SECI, Ba and Leadership: a unified model of dynamic knowledge creation. Long Range Planning, 33, 5-34; 2000.

NONAKA, I.; TAKEUCHI, H. Criação de Conhecimento na Empresa. 16. ed. Rio de Janeiro: Elsevier, 1997.

NONAKA, I.; VON KROGH, G; VOELPEL, S. Organizational knowledge creation theory: Evolutionary paths and future advances. Organization Studies, 179-1208, 2006.

NONAKA, I.; VON KROGH, G. Tacit Knowledge and Knowledge Conversion: Controversy and Advancement in Organizational Knowledge Creation Theory. Organization Science, 20, n.3, May-June, pp. 635-652, 2009.

NONAKA, I.; KONNO, N. The concept of "ba": Building a foundation for knowledge creation. California Management Review, 40, p. 40-54, 1998.

Perspectivas em Gestão \& Conhecimento, João Pessoa, v. 8, número especial, p. 70-85, out. 2018. 
REIS, R. S. Creative Process within Globally Distributed Teams: Brazil, Sweden, France and India. Management and Organizational Studies, v. 2, n. 1, p. 1-13, 2014.

RODRIGUES, I. Cultura e Desempenho de Equipes de Projetos Globais: Um Estudo em Empresas Multinacionais Brasileiras. [s.I.] Universidade de São Paulo - USP, 2010.

SCHEIN, Edgar H. Cultura organizacional e liderança. Tradução de Ailton Bonfim Brandão. São Paulo: Atlas, 2009, 441p.

SACKMANN, S. A.; FRIESL, M. Exploring cultural impacts on knowledge sharing behavior in project teams - results from a simulation study. Journal of Knowledge Management, v.11, $n$. 6, p.142.156, 2007.

SCHMIDMEIER, J. Dissertação: Desenvolvimento da competência intercultural grupal em equipes multiculturais de empresas multinacionais. [s.I.] UFPR, 2016.

SCHNECKENBERG, D.; TRUONG, Y.; MAZLOOMI, H. Microfoundations of innovative capabilities: The leverage of collaborative technologies on organizational learning and knowledge management in a multinational corporation. Technological Forecasting and Social Change, v. 100, p. 356-368, 2015.

STAKE, R. E. Pesquisa qualitativa: estudando como as coisas funcionam. Porto Alegre: Editora Penso, 2011.

TAYLOR, S.; OSLAND, J. S. The impact of intercultural communication on global organizational learning. In: Handbook of Organizational Learning and Knowledge Management. 2nd. ed. West-Sussex-UK: Blackwell-Willey, 2011.

VICENTE-OLIVA, S.; MARTÍNEZ-SÁNCHEZ, Á.; BERGES-MURO, L. Research and development project management best practices and absorptive capacity: Empirical evidence from Spanish firms. International Journal of Project Management, v. 33, n. 8, p. 1704-1716, 2015

VON KROGH, G.; ICHIJO, K.; NONAKA, I. Facilitando a Criação do Conhecimento: Reinventando a empresa com a inovação contínua. Rio de Janeiro: Campus, 2001.

YIN, R. K. Applications of case study research. Thousand Oaks (CA): SAGE, 1993.

ZOLLO, M.; WINTER, S. G. Deliberate Learning and the Evolution of Dynamics Capabilities. Organization Science, p. 47, 2002.

Artigo recebido em 17/10/2018 e aceito para publicação em 29/10/2018

Perspectivas em Gestão \& Conhecimento, João Pessoa, v. 8, número especial, p. 70-85, out. 2018. 